Acta Crystallographica Section E

Structure Reports

Online

ISSN 1600-5368

\section{1,1'-Dimethyl-4,4'-bipyridinium bis(triiodide)}

\section{Tuoping $\mathrm{Hu}$}

Department of Chemistry, North University of China, Taiyuan, Shanxi 030051 People's Republic of China

Correspondence e-mail: hutuopingsx@yahoo.com.cn

Received 3 March 2009; accepted 23 April 2009

Key indicators: single-crystal X-ray study; $T=296 \mathrm{~K}$; mean $\sigma(\mathrm{C}-\mathrm{C})=0.010 \AA$; $R$ factor $=0.040 ; w R$ factor $=0.073 ;$ data-to-parameter ratio $=28.8$.

In the title compound, $\mathrm{C}_{12} \mathrm{H}_{14} \mathrm{~N}_{2}{ }^{2+} \cdot 2 \mathrm{I}_{3}{ }^{-}$, the $1,1^{\prime}$-dimethyl- $4,4^{\prime}$ bipyridinium (DMBP) dication is charge balanced by two triiodide ions. The DMBP dication is planar within 0.010 (5) $\AA$. The asymmetric unit contains only half of the dication, the other half being generated by an inversion center. Weak $\mathrm{C}-\mathrm{H} \cdots \mathrm{I}$ interactions link the ions into sheets parallel to (121).

\section{Related literature}

For a dication with similar geometry, see: Russell \& Wallwork (1972). For anions with comparable geometry, see: Marsh (2004); Madsen et al. (1999).

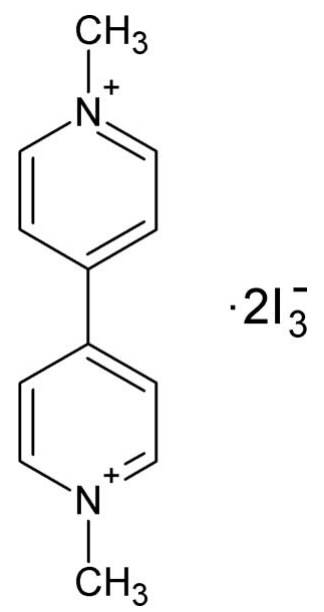

\section{Experimental}

\section{Crystal data}

$\mathrm{C}_{12} \mathrm{H}_{14} \mathrm{~N}_{2}{ }^{2+} \cdot 2 \mathrm{I}_{3}^{-}$

$M_{r}=947.65$

Triclinic, $P \overline{1}$

$a=7.5457$ (4) ^

$$
\begin{aligned}
& b=7.9541(6) \AA \\
& c=9.3029(6) \AA \\
& \alpha=90.306(5)^{\circ} \\
& \beta=94.192(4)^{\circ}
\end{aligned}
$$

$\gamma=102.332(5)^{\circ}$

$V=543.88(6) \AA^{3}$

$\mu=8.56 \mathrm{~mm}^{-1}$

$T=296 \mathrm{~K}$

$0.22 \times 0.16 \times 0.08 \mathrm{~mm}$

Mo $K \alpha$ radiation

Data collection

Bruker SMART CCD area-detector diffractometer

Absorption correction: multi-scan (SADABS; Sheldrick, 1996)

$T_{\min }=0.211, T_{\max }=0.504$

12956 measured reflections 2683 independent reflections 1468 reflections with $I>2 \sigma(I)$ $R_{\text {int }}=0.052$

Refinement

$R\left[F^{2}>2 \sigma\left(F^{2}\right)\right]=0.040$

$w R\left(F^{2}\right)=0.073$

$S=1.02$

2683 reflections

93 parameters

$\mathrm{H}$-atom parameters constrained

$\Delta \rho_{\max }=0.97 \mathrm{e} \AA^{-3}$

$\Delta \rho_{\min }=-0.86$ e $\AA^{-3}$

Table 1

Selected geometric parameters $\left(\AA{ }^{\circ}\right)$.

\begin{tabular}{llll}
\hline $\mathrm{I} 1-\mathrm{I} 2$ & $2.9341(8)$ & $\mathrm{I} 2-\mathrm{I} 3$ & $2.9061(8)$
\end{tabular}

I3-I2-I1 $177.49(2)$

Table 2

Hydrogen-bond geometry $\left(\AA,^{\circ}\right)$.

\begin{tabular}{lllll}
\hline$D-\mathrm{H} \cdots A$ & $D-\mathrm{H}$ & $\mathrm{H} \cdots A$ & $D \cdots A$ & $D-\mathrm{H} \cdots A$ \\
\hline $\mathrm{C} 3-\mathrm{H} 3 \cdots \mathrm{I} 3^{\mathrm{i}}$ & 0.93 & 3.05 & $3.951(8)$ & 163 \\
$\mathrm{C} 2-\mathrm{H} 2 \cdots \mathrm{I} 1^{\text {ii }}$ & 0.93 & 3.16 & $4.066(8)$ & 164 \\
$\mathrm{C} 5-\mathrm{H} 5 \cdots \mathrm{I} 2^{\mathrm{i}}$ & 0.93 & 3.13 & $3.839(7)$ & 135 \\
\hline
\end{tabular}

Symmetry codes: (i) $-x,-y+1,-z+1$; (ii) $-x+1,-y+1,-z+2$.

Data collection: SMART (Bruker, 2007); cell refinement: SAINTPlus (Bruker, 2007); data reduction: SAINT-Plus; program(s) used to solve structure: SHELXS97 (Sheldrick, 2008); program(s) used to refine structure: SHELXL97 (Sheldrick, 2008); molecular graphics: SHELXTL (Sheldrick, 2008); software used to prepare material for publication: SHELXTL.

The author is grateful for funding support from the Natural Science Foundation of Shanxi Province (2007011033), the Program of Technological Industrialization at the University of Shanxi Province (20070308) and the start-up fund of North University of China.

Supplementary data and figures for this paper are available from the IUCr electronic archives (Reference: EZ2167).

\section{References}

Bruker (2007). SMART and SAINT-Plus. Bruker AXS Inc., Madison, Wisconsin, USA.

Madsen, D., Burghammer, M., Fiedler, S. \& Müller, H. (1999). Acta Cryst. B55, 601-606.

Marsh, R. E. (2004). Acta Cryst. B60, 252-253.

Russell, J. H. \& Wallwork, S. C. (1972). Acta Cryst. B28, 1527-1533.

Sheldrick, G. M. (1996). SADABS. University of Göttingen, Germany.

Sheldrick, G. M. (2008). Acta Cryst. A64, 112-122. 


\section{supporting information}

Acta Cryst. (2009). E65, o1162 [doi:10.1107/S1600536809015207]

\section{1,1'-Dimethyl-4,4'-bipyridinium bis(triiodide)}

\section{Tuoping Hu}

\section{S1. Comment}

The title compound, (I), was obtained by chance when we tried to prepare the salt of the $\mathrm{Pb}(\mathrm{II})$ cation and DMBP in $\mathrm{MeOH}$. This paper provides the first crystal structure of the DMBP dication with two triiodide anions.

Only half of the dication of DMBP is contained in the asymmetric unit, while the other half is generated by the inversion center at (1/2,1/2,1/2) (Fig 1.). The $N, N^{\prime}$-dimethyl-4,4'bipyridylium(II) dication has an essentially planar conformation, the maximum deviation of the $\mathrm{C} 1$ atom (the methyl group) from its mean plane being 0.010 (5) $\AA$. The geometry of the dication is similar to the one observed in Russell \& Wallwork (1972). Meanwhile, the geometry of the anion is comparable to that described in Marsh (2004) and Madsen et al. (1999).

Weak $\mathrm{C} 3-\mathrm{H} 3 \cdots \mathrm{I} 3$ interactions link two $\mathrm{I}_{3}$ anions to each dication. A weaker $\mathrm{C} 2-\mathrm{I} 2 \cdots \mathrm{H} 1$ interaction links each anion to a further DMBP cation, to form sheets parallel to (121). Adjacent sheets are packed into a three-dimensional motif (Fig. 2).

\section{S2. Experimental}

$\mathrm{C}_{12} \mathrm{H}_{14} \mathrm{~N}_{2} .4 \mathrm{Cl}(0.5 \mathrm{mmol}, 128 \mathrm{mg})$ and $\mathrm{KI}(10 \mathrm{mmol}, 1660 \mathrm{mg})$ were added to $50 \mathrm{ml}$ of $\mathrm{CH}_{3} \mathrm{CN}$. After stirring and refluxing for $12 \mathrm{~h}$, the mixture was filtered, and the clear solution was allowed to evaporate slowly under inert atmosphere. Prismatic crystals of the title compound were obtained after 5 days. The crystals were filtered, washed by cool EtOH and dried in air.

\section{S3. Refinement}

All of the $\mathrm{H}$ atoms were positioned geometrically and refined using a riding model with $\mathrm{C}-\mathrm{H}=0.930 \AA$ and $0.96 \AA$, with $U_{\text {iso }}(\mathrm{H})=1.2$ and 1.5 times $U_{\text {eq }}(\mathrm{C})$, for aromatic and methyl hydrogens, respectively. 

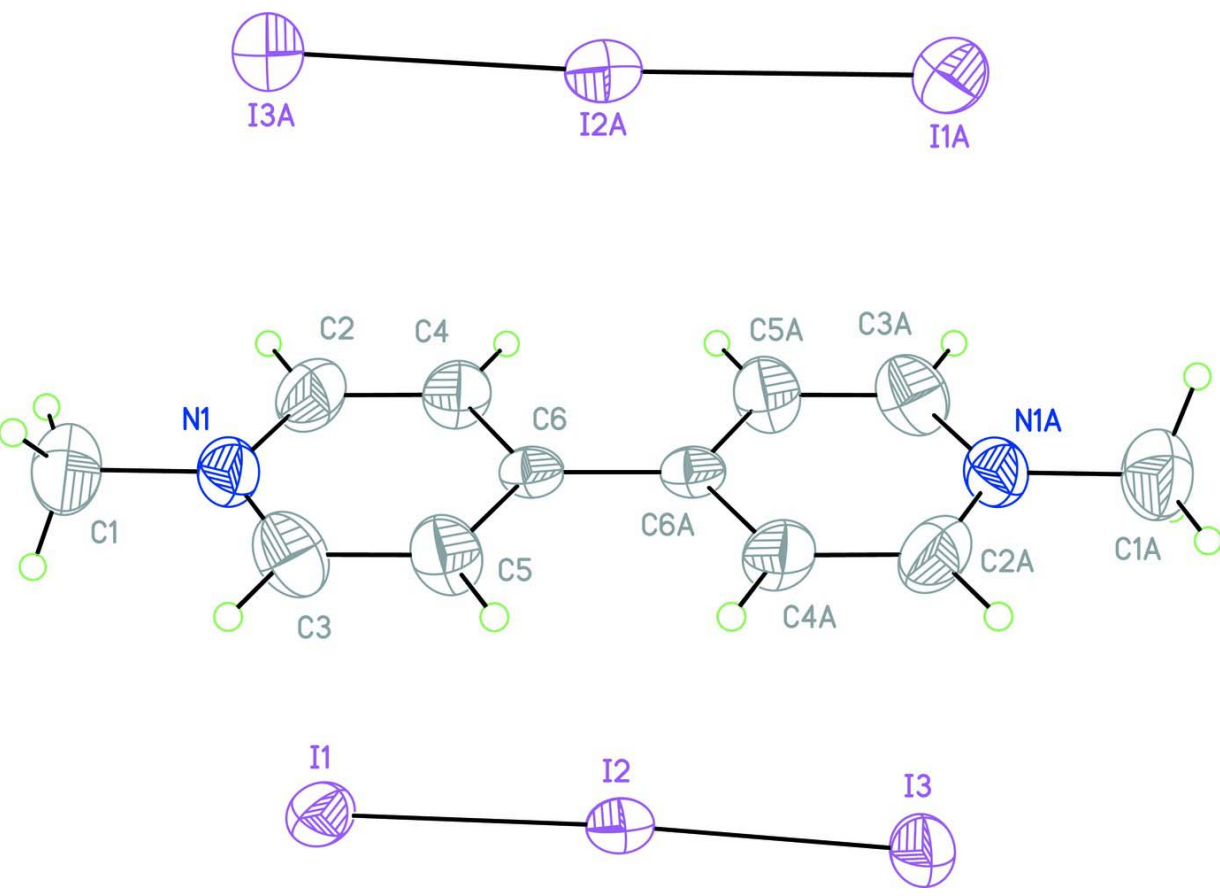

\section{Figure 1}

Molecular structure showing 50\% probability displacement ellipsoids. The atoms marked with A are derived from the reference atoms by means of the $(1-x, 1-y, 1-z)$ symmetry transformation..

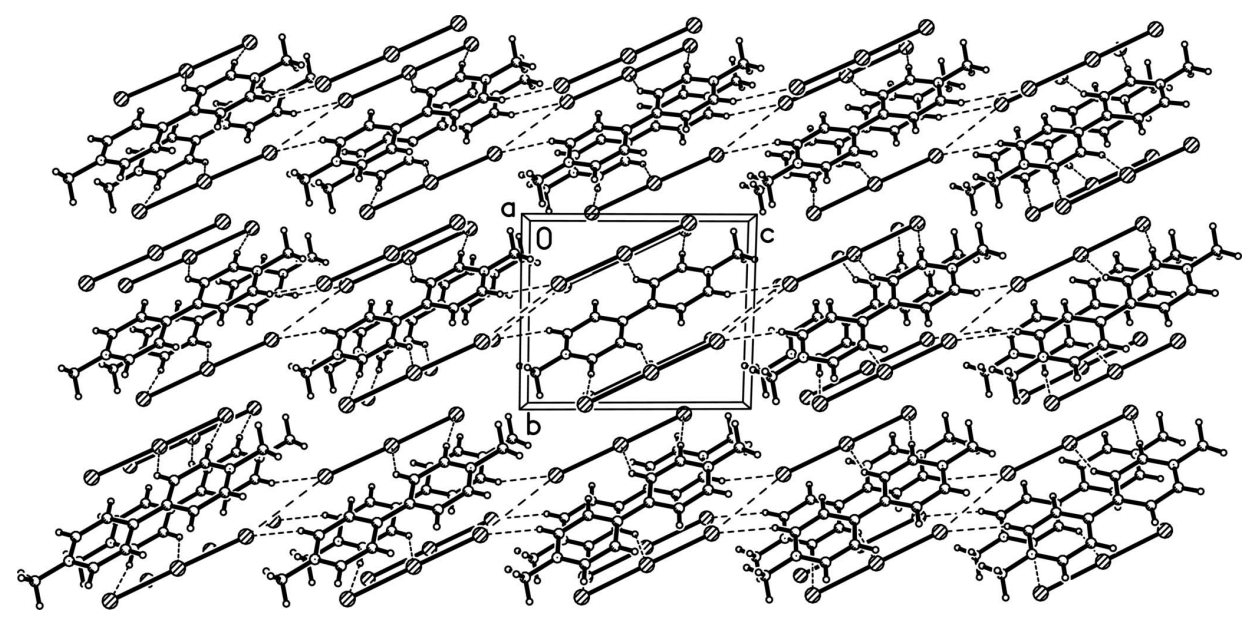

Figure 2

Packing diagram viewed down the $a$ axis. Weak C- $-\mathrm{H} \cdots \mathrm{I}$ interactions are shown as dotted lines.

\section{1,1'-Dimethyl-4,4'-bipyridinium bis(triiodide)}

Crystal data

$\mathrm{C}_{12} \mathrm{H}_{14} \mathrm{~N}_{2}{ }^{2+} \cdot 2 \mathrm{I}_{3}^{-}$

$M_{r}=947.65$

Triclinic, $P \overline{1}$

Hall symbol: -P 1

$a=7.5457$ (4) $\AA$

$b=7.9541(6) \AA$

$$
\begin{aligned}
& c=9.3029(6) \AA \\
& \alpha=90.306(5)^{\circ} \\
& \beta=94.192(4)^{\circ} \\
& \gamma=102.332(5)^{\circ} \\
& V=543.88(6) \AA^{3} \\
& Z=1
\end{aligned}
$$


$F(000)=418$

$D_{\mathrm{x}}=2.893 \mathrm{Mg} \mathrm{m}^{-3}$

Mo $K \alpha$ radiation, $\lambda=0.71073 \AA$

Cell parameters from 4412 reflections

$\theta=2.6-27.6^{\circ}$

\section{Data collection}

Bruker SMART CCD area-detector diffractometer

Radiation source: fine-focus sealed tube

Graphite monochromator

$\varphi$ and $\omega$ scans

Absorption correction: multi-scan

(SADABS; Sheldrick, 1996)

$T_{\min }=0.211, T_{\max }=0.504$

\section{Refinement}

Refinement on $F^{2}$

Least-squares matrix: full

$R\left[F^{2}>2 \sigma\left(F^{2}\right)\right]=0.040$

$w R\left(F^{2}\right)=0.073$

$S=1.02$

2683 reflections

93 parameters

0 restraints

Primary atom site location: structure-invariant direct methods

Secondary atom site location: difference Fourier map

\section{Special details}

Geometry. All e.s.d.'s (except the e.s.d. in the dihedral angle between two 1.s. planes) are estimated using the full covariance matrix. The cell e.s.d.'s are taken into account individually in the estimation of e.s.d.'s in distances, angles and torsion angles; correlations between e.s.d.'s in cell parameters are only used when they are defined by crystal symmetry. An approximate (isotropic) treatment of cell e.s.d.'s is used for estimating e.s.d.'s involving 1.s. planes.

Refinement. Refinement of $F^{2}$ against ALL reflections. The weighted $R$-factor $w R$ and goodness of fit $S$ are based on $F^{2}$, conventional $R$-factors $R$ are based on $F$, with $F$ set to zero for negative $F^{2}$. The threshold expression of $F^{2}>\sigma\left(F^{2}\right)$ is used only for calculating $R$-factors $(\mathrm{gt})$ etc. and is not relevant to the choice of reflections for refinement. $R$-factors based on $F^{2}$ are statistically about twice as large as those based on $F$, and $R$-factors based on ALL data will be even larger.

Fractional atomic coordinates and isotropic or equivalent isotropic displacement parameters $\left(\hat{A}^{2}\right)$

\begin{tabular}{lllll}
\hline & $x$ & $y$ & $z$ & $U_{\text {iso }} * / U_{\text {eq }}$ \\
\hline I1 & $0.11371(7)$ & $0.64766(7)$ & $0.84204(6)$ & $0.0705(2)$ \\
I2 & $0.19121(6)$ & $0.80427(6)$ & $0.56237(6)$ & $0.05927(17)$ \\
I3 & $0.25337(8)$ & $0.96496(8)$ & $0.28546(6)$ & $0.0816(2)$ \\
N1 & $0.3773(9)$ & $0.2800(7)$ & $0.8128(7)$ & $0.0588(16)$ \\
C1 & $0.3276(13)$ & $0.1852(11)$ & $0.9438(9)$ & $0.085(3)$ \\
H1A & 0.4248 & 0.2166 & 1.0179 & $0.128^{*}$ \\
H1B & 0.3064 & 0.0638 & 0.9235 & $0.128^{*}$ \\
H1C & 0.2190 & 0.2131 & 0.9758 & $0.128^{*}$ \\
C2 & $0.5358(12)$ & $0.3875(11)$ & $0.8116(9)$ & $0.074(2)$ \\
H2 & 0.6149 & 0.4034 & 0.8944 & $0.088^{*}$
\end{tabular}

Prism, black

$0.22 \times 0.16 \times 0.08 \mathrm{~mm}$

12956 measured reflections

2683 independent reflections

$R_{\text {int }}=0.052$

$\theta_{\max }=28.3^{\circ}, \theta_{\min }=3.9^{\circ}$

$h=-10 \rightarrow 10$

$k=-10 \rightarrow 10$

Hydrogen site location: inferred from eighbouring sites

where $P=\left(F_{\mathrm{o}}^{2}+2 F_{\mathrm{c}}{ }^{2}\right) / 3$

$(\Delta / \sigma)_{\max }<0.001$

$\Delta \rho_{\max }=0.97$ e $\AA^{-3}$
$\Delta \rho_{\min }=-0.86 \mathrm{e}^{-3}$

Extinction correction: SHELXL97 (Sheldrick, 2008), $\mathrm{Fc}^{*}=\mathrm{kFc}\left[1+0.001 \times \mathrm{Fc}^{2} \lambda^{3} / \sin (2 \theta)\right]^{-1 / 4}$

Extinction coefficient: 0.0028 (3) $\mu=8.56 \mathrm{~mm}^{-1}$ 


\begin{tabular}{lllll}
$\mathrm{C} 3$ & $0.2652(11)$ & $0.2566(10)$ & $0.6956(10)$ & $0.072(2)$ \\
$\mathrm{H} 3$ & 0.1526 & 0.1813 & 0.6969 & $0.086^{*}$ \\
$\mathrm{C} 4$ & $0.5864(9)$ & $0.4764(10)$ & $0.6903(8)$ & $0.061(2)$ \\
$\mathrm{H} 4$ & 0.6984 & 0.5532 & 0.6924 & $0.074^{*}$ \\
$\mathrm{C} 5$ & $0.3120(10)$ & $0.3414(10)$ & $0.5722(8)$ & $0.066(2)$ \\
$\mathrm{H} 5$ & 0.2309 & 0.3216 & 0.4906 & $0.079^{*}$ \\
$\mathrm{C} 6$ & $0.4743(8)$ & $0.4540(7)$ & $0.5658(7)$ & $0.0396(14)$ \\
\hline
\end{tabular}

Atomic displacement parameters $\left(\AA^{2}\right)$

\begin{tabular}{lllllll}
\hline & $U^{11}$ & $U^{22}$ & $U^{33}$ & $U^{12}$ & $U^{13}$ & $U^{23}$ \\
\hline $\mathrm{I} 1$ & $0.0689(4)$ & $0.0899(4)$ & $0.0576(3)$ & $0.0291(3)$ & $0.0010(3)$ & $0.0076(3)$ \\
$\mathrm{I} 2$ & $0.0503(3)$ & $0.0648(3)$ & $0.0672(3)$ & $0.0224(2)$ & $0.0040(2)$ & $0.0045(2)$ \\
$\mathrm{I} 3$ & $0.0838(4)$ & $0.0895(4)$ & $0.0816(4)$ & $0.0335(3)$ & $0.0267(3)$ & $0.0292(3)$ \\
$\mathrm{N} 1$ & $0.066(4)$ & $0.051(4)$ & $0.063(4)$ & $0.016(3)$ & $0.016(4)$ & $0.010(3)$ \\
C1 & $0.106(7)$ & $0.076(6)$ & $0.075(6)$ & $0.017(5)$ & $0.017(5)$ & $0.015(5)$ \\
C2 & $0.071(6)$ & $0.088(6)$ & $0.061(5)$ & $0.020(5)$ & $-0.011(4)$ & $0.015(5)$ \\
C3 & $0.062(5)$ & $0.066(5)$ & $0.078(6)$ & $-0.010(4)$ & $0.011(5)$ & $-0.003(5)$ \\
C4 & $0.039(4)$ & $0.077(5)$ & $0.058(5)$ & $-0.004(4)$ & $-0.016(3)$ & $0.006(4)$ \\
C5 & $0.052(5)$ & $0.080(6)$ & $0.054(5)$ & $-0.007(4)$ & $-0.003(4)$ & $-0.001(4)$ \\
C6 & $0.031(3)$ & $0.032(3)$ & $0.054(4)$ & $0.005(3)$ & $-0.002(3)$ & $-0.003(3)$ \\
\hline
\end{tabular}

Geometric parameters $\left(\stackrel{\AA}{\circ}{ }^{\circ}\right)$

\begin{tabular}{|c|c|c|c|}
\hline $\mathrm{I} 1-\mathrm{I} 2$ & $2.9341(8)$ & $\mathrm{C} 2-\mathrm{H} 2$ & 0.9300 \\
\hline $\mathrm{I} 2-\mathrm{I} 3$ & $2.9061(8)$ & $\mathrm{C} 3-\mathrm{C} 5$ & $1.364(10)$ \\
\hline $\mathrm{N} 1-\mathrm{C} 2$ & $1.314(9)$ & $\mathrm{C} 3-\mathrm{H} 3$ & 0.9300 \\
\hline $\mathrm{N} 1-\mathrm{C} 3$ & $1.317(9)$ & $\mathrm{C} 4-\mathrm{C} 6$ & $1.371(8)$ \\
\hline $\mathrm{N} 1-\mathrm{C} 1$ & $1.467(9)$ & $\mathrm{C} 4-\mathrm{H} 4$ & 0.9300 \\
\hline $\mathrm{C} 1-\mathrm{H} 1 \mathrm{~A}$ & 0.9600 & $\mathrm{C} 5-\mathrm{C} 6$ & $1.359(9)$ \\
\hline $\mathrm{C} 1-\mathrm{H} 1 \mathrm{~B}$ & 0.9600 & $\mathrm{C} 5-\mathrm{H} 5$ & 0.9300 \\
\hline $\mathrm{C} 1-\mathrm{H} 1 \mathrm{C}$ & 0.9600 & $\mathrm{C} 6-\mathrm{C}^{\mathrm{i}}$ & $1.464(12)$ \\
\hline $\mathrm{C} 2-\mathrm{C} 4$ & $1.370(10)$ & & \\
\hline $\mathrm{I} 3-\mathrm{I} 2-\mathrm{I} 1$ & $177.49(2)$ & $\mathrm{N} 1-\mathrm{C} 3-\mathrm{C} 5$ & $120.9(7)$ \\
\hline $\mathrm{C} 2-\mathrm{N} 1-\mathrm{C} 3$ & $119.7(7)$ & $\mathrm{N} 1-\mathrm{C} 3-\mathrm{H} 3$ & 119.5 \\
\hline $\mathrm{C} 2-\mathrm{N} 1-\mathrm{C} 1$ & $119.8(7)$ & $\mathrm{C} 5-\mathrm{C} 3-\mathrm{H} 3$ & 119.5 \\
\hline $\mathrm{C} 3-\mathrm{N} 1-\mathrm{C} 1$ & $120.5(7)$ & $\mathrm{C} 2-\mathrm{C} 4-\mathrm{C} 6$ & $121.0(6)$ \\
\hline $\mathrm{N} 1-\mathrm{C} 1-\mathrm{H} 1 \mathrm{~A}$ & 109.5 & $\mathrm{C} 2-\mathrm{C} 4-\mathrm{H} 4$ & 119.5 \\
\hline $\mathrm{N} 1-\mathrm{C} 1-\mathrm{H} 1 \mathrm{~B}$ & 109.5 & $\mathrm{C} 6-\mathrm{C} 4-\mathrm{H} 4$ & 119.5 \\
\hline $\mathrm{H} 1 \mathrm{~A}-\mathrm{C} 1-\mathrm{H} 1 \mathrm{~B}$ & 109.5 & $\mathrm{C} 6-\mathrm{C} 5-\mathrm{C} 3$ & $121.6(7)$ \\
\hline $\mathrm{N} 1-\mathrm{C} 1-\mathrm{H} 1 \mathrm{C}$ & 109.5 & $\mathrm{C} 6-\mathrm{C} 5-\mathrm{H} 5$ & 119.2 \\
\hline $\mathrm{H} 1 \mathrm{~A}-\mathrm{C} 1-\mathrm{H} 1 \mathrm{C}$ & 109.5 & $\mathrm{C} 3-\mathrm{C} 5-\mathrm{H} 5$ & 119.2 \\
\hline $\mathrm{H} 1 \mathrm{~B}-\mathrm{C} 1-\mathrm{H} 1 \mathrm{C}$ & 109.5 & $\mathrm{C} 5-\mathrm{C} 6-\mathrm{C} 4$ & $115.9(6)$ \\
\hline $\mathrm{N} 1-\mathrm{C} 2-\mathrm{C} 4$ & $121.0(7)$ & $\mathrm{C} 5-\mathrm{C} 6-\mathrm{C}^{\mathrm{i}}$ & $122.1(7)$ \\
\hline $\mathrm{N} 1-\mathrm{C} 2-\mathrm{H} 2$ & 119.5 & $\mathrm{C} 4-\mathrm{C} 6-\mathrm{C}^{\mathrm{i}}$ & $122.1(7)$ \\
\hline $\mathrm{C} 4-\mathrm{C} 2-\mathrm{H} 2$ & 119.5 & & \\
\hline
\end{tabular}




$\begin{array}{llll}\mathrm{C} 3-\mathrm{N} 1-\mathrm{C} 2-\mathrm{C} 4 & 0.3(12) & \mathrm{N} 1-\mathrm{C} 3-\mathrm{C} 5-\mathrm{C} 6 & -0.7(13) \\ \mathrm{C} 1-\mathrm{N} 1-\mathrm{C} 2-\mathrm{C} 4 & 179.4(7) & \mathrm{C} 3-\mathrm{C} 5-\mathrm{C} 6-\mathrm{C} 4 & 0.0(11) \\ \mathrm{C} 2-\mathrm{N} 1-\mathrm{C} 3-\mathrm{C} 5 & 0.6(12) & \mathrm{C} 3-\mathrm{C} 5-\mathrm{C} 6-\mathrm{C} 6 & -179.8(8) \\ \mathrm{C} 1-\mathrm{N} 1-\mathrm{C} 3-\mathrm{C} 5 & -178.5(7) & \mathrm{C} 2-\mathrm{C} 4-\mathrm{C} 6-\mathrm{C} 5 & 0.9(11) \\ \mathrm{N} 1-\mathrm{C} 2-\mathrm{C} 4-\mathrm{C} 6 & -1.0(12) & \mathrm{C} 2-\mathrm{C} 4-\mathrm{C} 6-\mathrm{C}^{\mathrm{i}} & -179.3(8)\end{array}$

Symmetry code: (i) $-x+1,-y+1,-z+1$.

Hydrogen-bond geometry $\left(\AA,{ }^{\circ}\right)$

\begin{tabular}{lllll}
\hline$D-\mathrm{H} \cdots A$ & $D-\mathrm{H}$ & $\mathrm{H} \cdots A$ & $D \cdots A$ & $D-\mathrm{H} \cdots A$ \\
\hline $\mathrm{C} 3-\mathrm{H} 3 \cdots \mathrm{I} 3^{\mathrm{ii}}$ & 0.93 & 3.05 & $3.951(8)$ & 163 \\
$\mathrm{C} 2-\mathrm{H} 2 \cdots \mathrm{I} 1^{\mathrm{iii}}$ & 0.93 & 3.16 & $4.066(8)$ & 164 \\
$\mathrm{C} 5-\mathrm{H} 5 \cdots \mathrm{I} 2^{\mathrm{ii}}$ & 0.93 & 3.13 & $3.839(7)$ & 135 \\
\hline
\end{tabular}

Symmetry codes: (ii) $-x,-y+1,-z+1$; (iii) $-x+1,-y+1,-z+2$. 